\title{
Design and Calibration of A 60-GHz Personal Exposimeter for Exposure Assessment in Specular and Diffuse Environments
}

\author{
Reza Aminzadeh ${ }^{1}$, Arno Thielens ${ }^{1}$, Abdou Khadir Fall ${ }^{2}$, Haolin $\mathrm{Li}^{1}$, \\ Carole Leduc ${ }^{3}$, Maxim Zhadobov ${ }^{3}$, Guy Torfs ${ }^{1}$, Johan Bauwelinck ${ }^{1}$, \\ Luc Martens ${ }^{1}$ and Wout Joseph ${ }^{1}$ \\ ${ }^{* 1}$ Department of Information Technology, Ghent University/iMinds \\ Technologiepark-Zwijnaarde 15, B-9052 Ghent, Belgium \\ ${ }^{2}$ Institute of Electronics and Telecommunications of Rennes (IETR), INSA, \\ UMR CNRS 6164, University of Rennes 1, 35708 Rennes, France \\ ${ }^{3}$ Institute of Electronics and Telecommunications of Rennes (IETR) \\ UMR CNRS 6164, University of Rennes 1, 35042 Rennes, France
}

\begin{abstract}
For the first time, a personal exposimeter (PE) for $60 \mathrm{GHz}$ radiation measurements is presented. The PE is designed based on numerical simulations, onphantom and on-body calibration measurements under specular and diffuse exposure to determine the antenna aperture and measurement uncertainty of the PE. A 95\% prediction interval of $6.6 \mathrm{~dB}$ is obtained for the PE's response using a combination of three nodes with different polarizations. The response of the $\mathrm{PE}$ is in the range of 0.7 to $\mathbf{1 . 2}$ for specular and diffuse fields.
\end{abstract}

\section{INTRODUCTION}

Recent developments in $60-\mathrm{GHz}$ communication systems have heightened the need for assessing potential health effects of mm-waves [1]. Research on biological effects of mm-waves is necessary to ensure safety of mm-wave systems and to update the related safety standards. Before being commercialized, any wireless device should comply with international guidelines, such as those issued by the International Commission on Non-Ionizing Radiation Protection (ICNIRP) [2]. The incident power density (IPD) is used as a dosimetric quantity at $60-\mathrm{GHz}$ [2].

Previous dosimetric studies in the $60 \mathrm{GHz}$ band are not yet widely established on a real human subject [1], [3]. Therefore, assessment of human exposure to mm-wave systems in real life scenarios is necessary.

A large number of studies, for example [4]-[6], have investigated everyday life IPD measurements using personal exposimeters (PEMs) for frequencies lower than $6 \mathrm{GHz}$. PEMs measure the total electric fields near the body instead of the actual incident fields, and are consequently faced with large measurement uncertainties [7].

People spend most of their time indoor and are exposed to EM radiation continuously. According to the room electromagnetics' theory [8], the total power in an indoor environment is composed of specular and diffuse multipath components (DMC), which result from coherent and non-coherent reflections (multiple sets of diffracted waves), respectively.

In this paper, for the first time, a prototype of wearable $60-\mathrm{GHz}$ on-body personal exposimeter (PE) is presented for specular and diffuse fields exposure. The PE consists of three receiving nodes (antennas) to measure the IPD in realistic indoor environments, and is calibrated in anechoic conditions using a real human subject and in a mm-wave reverberation chamber (RC) on a skin-equivalent phantom. The measurement uncertainty of the proposed prototype of $\mathrm{PE}$ is assessed in terms of numerical simulations and calibration measurements.

\section{MATERIALS AND METHODS}

First, the design of the PE which is based on numerical simulations at $60 \mathrm{GHz}$, is described in Section II-A. Next, the on-body calibrations on a real human subject (in anechoic conditions) and on two skin-equivalent phantoms (in anechoic conditions and in an RC) are described in Section II-B. The processing of the measurements is described in Section II-B3.

\section{A. numerical modeling}

Figure 1 illustrates the proposed numerical model of a PE. The finite-difference time-domain (FDTD) simulation platform, Sim4Life [9], is used to determine the distribution of the on-body received power on the antenna. A homogeneous skin model $\left(200 \times 200 \times 10 \mathrm{~mm}^{3}\right)$ is used during simulations to emulate the human body. The dielectric properties of this model are taken from dry skin at $60 \mathrm{GHz}$ [10]. 
The antenna used for modeling (see Figure 1) is a microstrip-fed antenna array, operating in the 60$\mathrm{GHz}$ band [11].

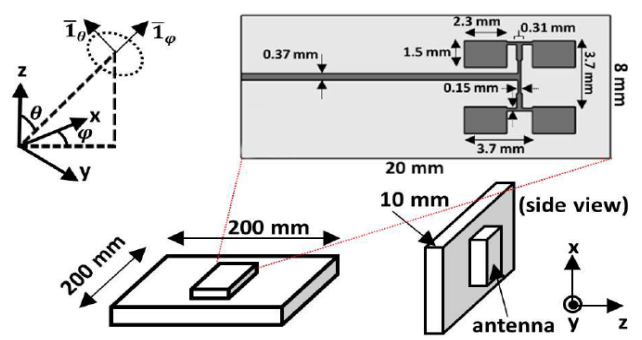

Fig. 1. The proposed model for numerical simulations. Schematic representation of a single node (antenna) at $5.6 \mathrm{~mm}$ from the skin used for simulations and measurements [11].

Simulations are performed for the antenna in free space and near the skin model $(5.6 \mathrm{~mm}$ due to the geometry of connector) to determine the response of the antenna. The response $(R)$ of the antenna is studied as the ratio of the median received power onbody $\left(P_{r}^{b o d y}\right)$ to the median received power in free space $\left(P_{r}^{\text {free }}\right)$ :

$$
R=\frac{P_{r}^{\text {body }}}{P_{r}^{\text {free }}}
$$

The received power on an antenna can be determined from its aperture [12]:

$$
P_{r}(\varphi, \theta)=A A(\varphi, \theta) \times S_{i n c}
$$

where $A A$ and $S_{i n c}$ are the on-body antenna aperture and incident power density, respectively. In order to calculate the on-body aperture of the antenna $A A(\varphi, \theta)$, the directive gain of the antenna $D(\varphi, \theta)$ is extracted for azimuth angle $(\varphi)$ and polar angle $(\theta)$ from numerical simulations as:

$$
A A(\varphi, \theta)=\eta_{\text {rad }}\left(1-\left|S_{11}\right|^{2}\right) D(\varphi, \theta) \frac{\lambda^{2}}{4 \pi}
$$

where $\eta_{\text {rad }}$ is the radiation efficiency, $\left|S_{11}\right|^{2}$ is the antenna's power reflection coefficient, and $\lambda$ is the wavelength. $A A(\varphi, \theta)$ can be determined for two orthogonal polarizations on the incident electric fields: $\theta$ and $\varphi$, which are the polarizations parallel to the unity vectors $\overline{1}_{\theta}$ and $\overline{1}_{\phi}$.

For the specular fields, a realistic far-field exposure scenario in the $60-\mathrm{GHz}$ band for an indoor environment (IEEE 802.11 standard [13]) is considered to determine the response of the simulated on-body antenna. A Gaussian distribution with a cross polarization ratio of $20 \mathrm{~dB}$ is assumed for the indoor exposure scenario [13]. The phase $(\alpha)$ of plane waves is assumed to have a uniform distribution in the range of $0<\psi<2 \pi$. For $\theta$ and $\varphi$, samples are drawn from their distributions [13].
For diffuse fields $\alpha, \varphi, \theta$ and $\psi$ are all assumed to have a uniform distribution [8].

\section{B. calibration measurement setup}

The calibration measurements are performed in anechoic conditions for specular fields (Section II-B1) and in a reverberation chamber for diffuse fields (Section II-B2).

1) on-body calibration: specular fields: Figure 2 demonstrates the measurement setup (at $60 \mathrm{GHz}$ ) for on-body calibration in specular fields. A standard gain horn antenna (19 $\mathrm{dBi})$ is used as transmitter (TX) and is mounted on a rotation stage (see right hand side of Figure 2). The receiver (RX)-the same antenna used in simulations-is placed in the far field of the TX. An Agilent PNA-X N5247A network analyzer delivers a constant power $(6 \mathrm{dBm})$ to the TX and measures the received on-body power on the RX. Two orthogonal polarizations of TX (to account for any realistic polarization) and RX (due to the asymmetric shape of RX) are studied.

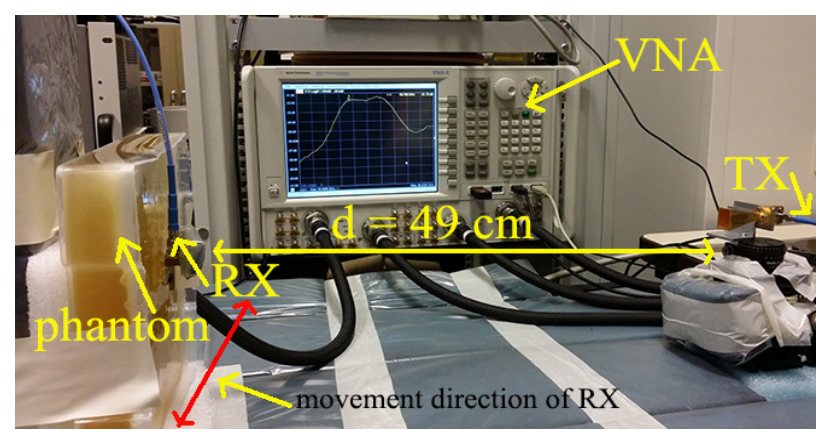

Fig. 2. Measurement setup in anechoic conditions at $60 \mathrm{GHz}$.

First, the free-space incident power density $S_{i n c}^{\text {free }}$ is calculated using the Friis formula [12] and then is averaged over $20 \mathrm{~cm}^{2}$ of the studied area [2].

Second, the RX is placed horizontally on a skinequivalent phantom (30\% gelatin, $70 \%$ water) following the instructions of [14]. The received power on the RX is calculated from the measured power gain $\left(\left|S_{21}\right|^{2}\right)$ for five azimuth angles $(\varphi)$ : $-20^{\circ}$ to $23^{\circ}$.

Third, the above step is repeated for two polarizations of TX and RX by placing the RX on the forearm of a male subject.

In the fourth step, three locations are selected for the RX on the subject's forearm and the received power is measured on each RX. Next, the best combination of three antennas on the forearm is determined based on the $50 \%$ prediction interval $\left(\mathrm{PI}_{50}\right)$ of the measurements (see Figure 3).

2) on-body calibration: diffuse fields: Figure 4 shows the measurement setup in the reverberation chamber at $60 \mathrm{GHz}$. An RC with $0.58 \times 0.592 \times 0.595 \mathrm{~m}^{3}$ dimensions is used. A 


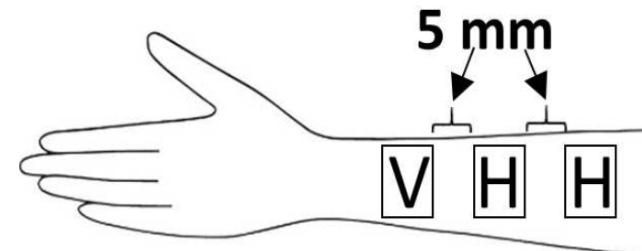

Fig. 3. The optimized orientation of the antennas on the subject's forearm determined from the on-body calibration measurements (not to scale).

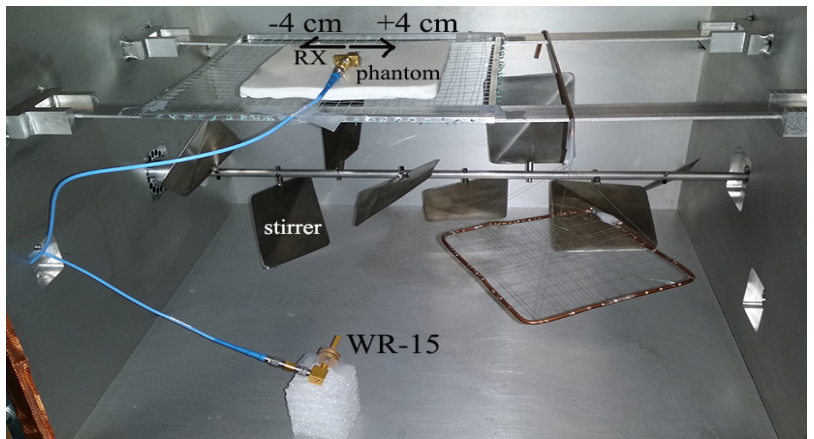

Fig. 4. Measurement setup in RC at $60 \mathrm{GHz}$. Three locations of RX is indicated on top of the phantom. ( \pm is used to indicate right and left.)

Wiltron 360 VNA is used to feed a rectangular WR15 waveguide as the source (TX) and to measure the scattering parameters of RX for 100 positions of the stirrer during a full rotation. A semi-solid phantom is used to emulate the dielectric properties of human skin at $60 \mathrm{GHz}$ [15].

Firstly, scattering parameters are measured in free space in the RC. For the antenna on body, the lossy phantom can load the chamber during calibration measurements and thus will change the power density values inside the chamber [16]. Therefore, the phantom is placed far away from the RX during the free space measurements.

Secondly, the RX is placed on the center of the phantom and the scattering parameters are measured on three locations of RX on the phantom (see Figure 4; center and $4 \mathrm{~cm}$ from center on the right and the left).

The antenna aperture is calculated from the Qfactor [17]. Therefore, following equations(1) and (2), the response (the ratio of antenna aperture) for diffuse fields $R_{R C}$ is defined as:

$$
R_{R C}=\frac{A A_{\text {phantom }}}{A A_{\text {free-space }}}=\frac{\left\langle\left|S_{21, p h}\right|^{2}-\left|\left\langle S_{21, p h}\right\rangle\right|^{2}\right\rangle}{\left\langle\left|S_{21, f r}\right|^{2}-\left|\left\langle S_{21, f r}\right\rangle\right|^{2}\right\rangle}
$$

where $S_{21, p h}$ and $S_{21, f r}$ are S-parameters for the $\mathrm{RX}$ on the phantom and the RX in free space (phantom at a far distance from the RX), respectively.
The notation \langle\rangle stands for an average over the stirrer's positions.

3) process measurements in a real environment: During the measurements in the real environment a power $\left(P_{r, i}^{\text {meas }}\right)$ is recorded on each antenna $i$. This received power can be used to determine the incident power density $\left(S_{i n c}\right)$ :

$$
S_{i n c}=\frac{P_{r, i}^{\text {meas }}}{A A_{i}}
$$

where $A A_{i}\left(\mathrm{~m}^{2}\right)$ is the effective median on-body antenna aperture obtained from calibration measurements. For specular fields, $A A_{i}(\varphi, \psi)$ values are determined for any realistic polarization (as the the sum of two orthogonal polarizations) [18]:

$$
\begin{aligned}
A A_{i}(\varphi, \psi) & =\frac{P_{r, i}^{b o d y, H}(\varphi)}{S_{i n c}^{f r e e, H}} \cos ^{2}(\psi) \\
& +\frac{P_{r, i}^{b o d y, V}(\varphi)}{S_{i n c}^{\text {free }, V}} \sin ^{2}(\psi)
\end{aligned}
$$

The free-space power density $S_{i n c}^{f r e e, H / V}$ for the same input power is obtained from the first step of calibration measurements. A Gaussian distribution that is applicable for indoor $60 \mathrm{GHz}$ communications is used for specular fields [13]. For diffuse fields a uniform distribution is used [8]. During the calibration measurements the powers $P_{r, i}^{b o d y, H / V}(\varphi)$ are registered for five angles $(\varphi)$. These antenna apertures $A A_{i}$ are determined for a realistic polarization $\psi$. Next, $1000 \psi$-samples are generated for every measured power value and this is repeated during 1000 iterations. Calculating $A A_{i}$, for every pair of $(\varphi, \psi)$, results in a distribution of $A A_{i}$ for every antenna $i$.

For diffuse fields, $R_{R C}$ values are used in equation (5) to calculate the incident power density on body.

\section{RESULTS AND DISCUSSION}

\section{A. specular fields}

Fig. 5 shows the distribution of the on-body antenna aperture obtained from calibration measurements for both $\mathrm{H}$ and $\mathrm{V}$ polarizations of the TX and RX. The measured antenna aperture for the horizontally $(\mathrm{H})$ oriented $\mathrm{RX}$ on-body and on the skin equivalent phantom are also in good agreement (difference is only $8.3 \%$ for the median values). These data are in good agreement with the antenna aperture determined from simulations (difference of $17.7 \%$ for the median values). The median antenna aperture $A A_{i}$ is $0.1 \mathrm{~mm}^{2}$ (horizontal orientation of $\mathrm{RX}$ ) on phantom, $0.092 \mathrm{~mm}^{2}$ (horizontal RX) on 


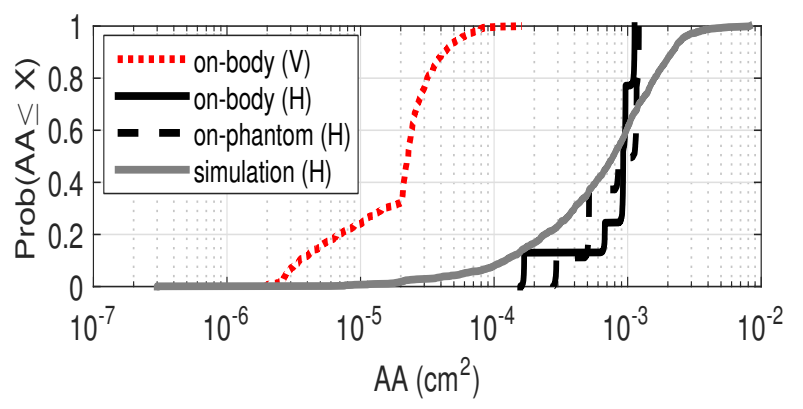

Fig. 5. Distribution of the on-body antenna aperture: calibration measurements vs. simulations.

body, $0.077 \mathrm{~mm}^{2}$ (horizontal $\mathrm{RX}$ ) for simulations and $23 \times 10^{-3} \mathrm{~mm}^{2}$ (vertical RX) on body. Thus, the results suggest that the absorbed energy for the body (horizontal RX) is higher than the phantom, which leads to a lower received power on the antenna on body and thus a lower AA value for the body with respect to the phantom.

The response is determined using (1) and is equal to $0.8(-0.96 \mathrm{~dB}), 0.75(-1.25 \mathrm{~dB})$ and $0.72(-1.4 \mathrm{~dB})$ for one-antenna calibration measurements on body, measurements on the phantom and for simulations on the phantom, respectively. This indicates underestimation of the incident electric fields with respect to free space values. This conclusion is also obtained for exposure assessment at frequencies $\leq 6 \mathrm{GHz}[6]$, [7].

\section{B. diffuse fields}

Figure 6 shows the ratio of AA (response) for diffuse fields. At $60 \mathrm{GHz}$ the $R_{R C}$ is equal to 1.2 which is higher than the calculated response for specular fields $(0.75$ for the phantom at $60 \mathrm{GHz})$. The value of $R_{R C}$ changes in the range of 1-1.2 and is reduced to about 0.9 with increasing the frequency. The simulated response for the diffuse exposure is obtained as 0.96 at $60 \mathrm{GHz}$ and is $22.2 \%$ different from the measured value at the same frequency.

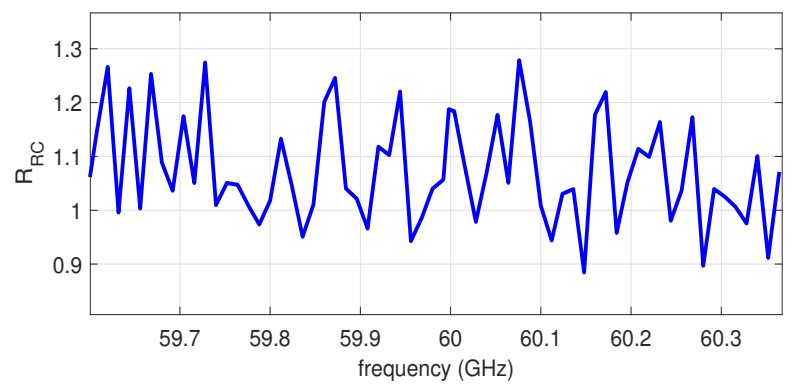

Fig. 6. Ratio of antenna aperture in RC.

\section{C. multi-node design of antennas}

Fig. 7 compares the $\mathrm{PI}_{50}$ and $\mathrm{PI}_{95}$ of the angular averaged response $\left(\mathrm{R}_{\text {meas }}\right)$ of the PE consisting of 1 ,
2 and 3 antennas for the specular fields, according to the best combination of three antennas on the subject's forearm. The value of $\mathrm{PI}_{50}$ is reduced from $4.4 \mathrm{~dB}$ to $3.6 \mathrm{~dB}$ and $1.3 \mathrm{~dB}$ for three, two and one antenna, respectively. Thus an improvement of $3.1 \mathrm{~dB}$ is obtained for three antennas vs. one antenna. Our proposed exposimeter has an improved $\mathrm{PI}_{50}$ of $16.6 \mathrm{~dB}$ ( 1 antenna) and $19.7 \mathrm{~dB}$ (3 antennas) compared to the $\mathrm{PI}_{50}$ of commercial PEMs (up to $21 \mathrm{~dB}$ ) at lower frequencies [7]. Also the $\mathrm{PI}_{95}$ value is reduced from $15.4 \mathrm{~dB}$ (1 antenna) to $6.6 \mathrm{~dB}(2$ and 3 antennas), which is an $8.8 \mathrm{~dB}$ improvement.

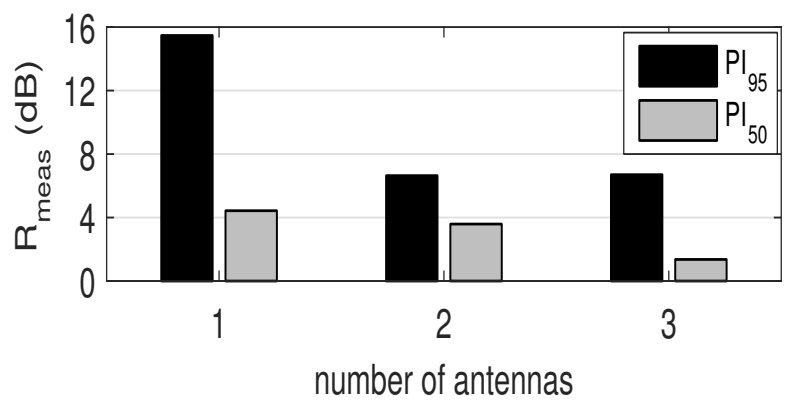

Fig. 7. Angular averaged response with smallest 50\% and $95 \%$ prediction intervals obtained from on-body calibration measurements.

\section{CONCLUSION}

A personal exposimeter is proposed using a limited number of wearable antennas in the $60 \mathrm{GHz}$ band. Using a combination of multiple nodes, the proposed exposimeter showed good accuracy. An improvement of 3-9 dB is obtained for three antennas compared to a single antenna experiment. The proposed prototype is the first $60 \mathrm{GHz} \mathrm{PE}$ and to the authors' best knowledge no data are available in the literature. Future work will consist of developing the PE for an improved wearability and multiple nodes over the user's body as well as a real measurement campaign in the $60 \mathrm{GHz}$ band.

\section{ACKNOWLEDGMEnTs}

This work was supported by the Research Foundation - Flanders (FWO-V) under grant agreement No G027714N.

\section{REFERENCES}

[1] T. Wu, T.S. Rappaport, and C.M. Collins. Safe for generations to come: Considerations of safety for millimeter waves in wireless communications. IEEE Microw. Mag., 16(2):65-84, March 2015.

[2] International Commission on Non-ionizing Radiation Protection (ICNIRP). Guidelines for limiting exposure to timevarying electric, magnetic, and electromagnetic fields (up to $300 \mathrm{GHz}$ ). Health Phys., 74(4):494-522, 1998. 
[3] M. Zhadobov, R. Sauleau, R. Augustine, C. Le Quément, Y. Le Dréan, and D. Thouroude. Near-field dosimetry for in vitro exposure of human cells at $60 \mathrm{GHz}$. Bioelectromagnetics, 33(1):55-64, 2012.

[4] J.F. Bolte and T. Eikelboom. Personal radiofrequency electromagnetic field measurements in the netherlands: Exposure level and variability for everyday activities, times of day and types of area. Environment International, 48:133 $-142,2012$.

[5] A. Thielens, S. Agneessens, L. Verloock, E. Tanghe, H. Rogier, L. Martens, and W. Joseph. On-body calibration and processing for a combination of two radio-frequency personal exposimeters. Radiat Prot Dosimetry, 163(1):5869, 2015.

[6] R. Aminzadeh, A. Thielens, A. Bamba, L. Kone, D.P. Gaillot, M. Lienard, L. Martens, and W. Joseph. On-body calibration and measurements using personal radiofrequency exposimeters in indoor diffuse and specular environments. Bioelectromagnetics, 37(5):298-309, 2016.

[7] J.F. Bolte, G. van der Zande, and J. Kamer. Calibration and uncertainties in personal exposure measurements of radiofrequency electromagnetic fields. Bioelectromagnetics, 32(8):652-663, 2011.

[8] J. B. Andersen, J. O. Nielsen, G. F. Pedersen, G. Bauch, and J. M. Herdin. Room electromagnetics. IEEE Antennas and Propagation Magazine, 49(2):27-33, April 2007.

[9] Speag. Sim4Life Reference Manual, 2016. http://www.zurichmedtech.com/sim4life/.

[10] S. Gabriel, R.W. Lau, and C. Gabriel. The dielectric properties of biological tissues: III. parametric models for the dielectric spectrum of tissues. Phys. Med. Biol., 41, 1996.

[11] N. Chahat, M. Zhadobov, L. Le Coq, S.I. Alekseev, and R. Sauleau. Characterization of the interactions between a $60-\mathrm{GHz}$ antenna and the human body in an off-body scenario. IEEE Trans. Antennas Propag., 60(12):59585965, Dec 2012.
[12] C. A. Balanis. Antenna Theory: Analysis and Design. Wiley-Interscience, 3 edition, 2005.

[13] A. Maltsev, V. Erceg, E. Perahia, Ch. Hansen, R. Maslennikov, A. Lomayev, A. Sevastyanov, A. Khoryaev, G. Morozov, M. Jacob, S. Priebe, Th. Kürner, Sh. Kato, H. Sawada, K. Sato, and H. Harada. Channel models for $60 \mathrm{GHz}$ WLAN Systems. Technical Report IEEE 802.1109/0334r8, IEEE, May 2010.

[14] R. Aminzadeh, M. Saviz, and A.A. Shishegar. Theoretical and experimental broadband tissue-equivalent phantoms at microwave and millimetre-wave frequencies. Electronics Letters, 50(8):618-620, April 2014.

[15] N. Chahat, M. Zhadobov, and R. Sauleau. Broadband tissue-equivalent phantom for ban applications at millimeter waves. IEEE Trans. Microw. Theory Techn., 60(7):2259-2266, July 2012.

[16] A. K. Fall, P. Besnier, C. Lemoine, M. Zhadobov, and R. Sauleau. Experimental dosimetry in a mode-stirred reverberation chamber in the $60-\mathrm{GHz}$ band. IEEE Transactions on Electromagnetic Compatibility, 58(4):981-992, Aug 2016.

[17] Philippe Besnier, Christophe Lemoine, and Jerome Sol. Various estimations of composite q-factor with antennas in a reverberation chamber. In 2015 IEEE International Symposium on Electromagnetic Compatibility (EMC), pages 1223-1227. IEEE, 2015.

[18] A. Thielens, S. Agneessens, H. De Clercq, J. Lecoutere, L. Verloock, E. Tanghe, S. Aerts, R. Puers, H. Rogier, L. Martens, and W. Joseph. On-body calibration and measurements using a personal, distributed exposimeter for wireless fidelity. Health Phys., 108(4):407-418, 2015. 